\title{
THE CUBIC NON-LINEARITY OF ORDER $\varepsilon$ IN THE SECOND APPROXIMATION
}

\author{
NGUYEN VAN DINH \\ Institute of Mechanics
}

It is well-known that, for almost quasi-linear systems, the oscillation can qualitatively and quantitatively - be determined in the first approximation (order $\varepsilon)$. However, for certain systems and even for qualitative information, the calculus must be performed in the second approximation (order $\varepsilon^{2}$ ). Some of mentioned systems have been considered in $[1,3,3]$.

Especially, in $[4,5,6,7]$, a systematic study has been devoted to a whole class of systems that having the quadratic restoring non-linearity as an element of order $\varepsilon$. There. the author has particularly concentrated attention on the effect of the quadratic non-linearity in the second approximation. It has been shown that although, in the first approximation, the non-linearity interested does not express any influence (on a family of harmonic oscillations with arbitrary constant amplitude and initial dephase and with frequency equal to the own frequency) it may play an important-even decisive - role in the second approximation. For instance, under certain condition (very weak friction) and in "combination" with certain other elements (excitations) of the same order $\varepsilon$, the quadratic non-linearity may produce intense oscillations of parametric type.

In the present paper, intending to develop the results obtained in $[4,5,6$, 7 ], we examine the class of systems with the cubic restoring non-linearity. This non-linearity introduces a lot of terms into the equations for stationary oscillations and makes thus difficulty revealing its own effects. In order to analyse the role of the cubic nonlinearity in the second approximation, we use the so-called simplified equations obtained by estimating and neglecting certain terms of order higher than $\varepsilon^{2}$.

The asymptotic method [8] is applied. Typical system are treated and some qualitative remarks are presented. 


\section{$\S 1$. The cubic non-linearity and a self-excitation of order $\varepsilon$}

In this section, we consider a self-excited system of VanderPol type assuming that the self-excitation is of the same order $\varepsilon$ as that of the cubic non-linearity. It will be shown that:

In the first approximation, the cubic nonlinearity introduces an additional frequency of order $\varepsilon$ but it has no influence on the amplitude (the latter is determined by the self-excitation).

In the second approximation, the corresponding additional frequency is negligible while the additional amplitude is of order $\varepsilon$ and significant. The system under consideration is described by the differential equation:

$$
\ddot{x}+x=\varepsilon\left\{h\left(1-k x^{2}\right) \dot{x}-\gamma x^{3}\right\},
$$

where $x$ is an oscillatory variable, overdots denote derivation with respect to time $t$; 1 is the own-frequency; $\varepsilon$ is a small positive formal parameter; $h>0$ and $k>0$ are coefficients characterizing the self-exciting mechanism; $\gamma$ is the cubic non-linearity coefficient.

Following the asymptotic method [8], the solution in the first approximation is of the form:

$$
\begin{array}{ll}
x=a \cos \psi, & \psi=t+\theta, \\
\dot{a}=\varepsilon A_{1}(a), & \dot{\theta}=\varepsilon B_{1}(a) .
\end{array}
$$

Substituting (1.2) into (1.1), using (1.3), equating the terms of like power in $\varepsilon$ then the ones of same harmonics in $\psi$, we obtain:

$$
\left\{\begin{array}{l}
\dot{a}=\varepsilon A_{1}(a)=\varepsilon \frac{h a}{2}\left(1-\frac{k}{4} a^{2}\right), \\
\dot{\theta}=\varepsilon B_{1}(a)=\varepsilon \frac{3 \gamma}{8} a^{2} .
\end{array}\right.
$$

Thus, in the first approximation, we have a family of harmonic oscillations with determined (not arbitrary) amplitude $a_{0}^{2}=\frac{4}{k}$, with arbitrary initial dephase $\theta_{0}$ and with frequency

$$
\dot{\psi}=\omega+\dot{\theta}=1+\varepsilon \frac{3 \gamma}{8} a_{0}^{2}=1+\varepsilon \frac{3 \gamma}{2 k}
$$

(thus, there is an additional frequency $\frac{3 \gamma}{2 k}$ of order $\varepsilon$, due to the cubic non-linearity). 
In the second approximation, the solution is determined from:

$$
\begin{gathered}
x=a \cos \psi+\varepsilon u_{1}(a, \psi)=a \cos \psi+\varepsilon\left(\frac{\gamma a^{3}}{32} \cos 3 \psi-\frac{h k a^{3}}{32} \sin 3 \psi\right) \\
\left\{\begin{array}{c}
\dot{a}=f=\varepsilon A_{1}(a)+\varepsilon^{2} A_{2}(a)= \\
=\varepsilon \frac{h a}{2}\left(1-\frac{k a^{2}}{4}\right)-\frac{\varepsilon^{2}}{2}\left\{2 A_{1} B_{1}+a A_{1} \frac{\partial A_{1}}{\partial a}+\gamma h a^{3}\left(\frac{k}{4} a^{2}-1+\frac{5}{8}\right)\right\}, \\
\dot{\theta}=\varepsilon B_{1}(a)+\varepsilon^{2} B_{2}(a)= \\
=\varepsilon \frac{3 \gamma}{8} a^{2}-\frac{\varepsilon^{2}}{2 a}\left\{-A_{1} \frac{\partial A_{1}}{\partial a}+h A_{1}-\frac{3}{4} h k a^{2} A_{1}+\frac{h^{2} k^{2}+15 \gamma^{2}}{128} a^{5}\right\} .
\end{array}\right.
\end{gathered}
$$

Evidently, even in the second approximation, we also obtain a family of oscillations whose "major" amplitude $a$ is given by the equation

$$
f=0 .
$$

The structure of this equation shows that $A_{1}(a)$ must be of order $\varepsilon^{2}$ i.e. $\left(1-\frac{k}{4} a^{2}\right)$ must be of order $\varepsilon$ (since $h$ is also of order $\varepsilon$ ). So, we can neglect the terms $2 A_{1} B_{1}$, $a A_{1} \frac{\partial A_{1}}{\partial a}$ and $\gamma h a^{2}\left(\frac{k a^{2}}{4}-1\right)$ (which are of order $\varepsilon^{3}$ ) and obtain a more simple equation

$$
f=\frac{h a}{2}\left(1-\frac{k a^{2}}{4}\right)-\frac{5 \gamma h a^{3}}{16}=0 .
$$

The amplitude is now equal to

$$
a_{*}^{2}=\frac{4}{k+\frac{5 \gamma}{2}} \approx a_{0}^{2}\left(1-\varepsilon \frac{5 \gamma}{2 k}\right)=a_{0}^{2}-\varepsilon \frac{10 \gamma}{k^{2}},
$$

and the dephase $\theta$ is

$$
\theta=\left\{\frac{3 \gamma}{8} a_{*}^{2}-\frac{a_{*}^{4}}{256}\left(h^{2} k^{2}+15 \gamma^{2}\right)\right\} t+\theta_{0}
$$

where $\theta_{0}$ is the arbitrary initial dephase.

We see that, in the second approximation, the effects of the cubic non-linearity consists of

- a perturbation $\frac{\gamma a^{3}}{32} \cos 3 \psi$ of order $\varepsilon$

- an additional amplitude $-\varepsilon \frac{10 \gamma}{k^{2}}$ of order $\varepsilon$. 
- a negligible additional frequency $-\frac{15 \gamma^{2} a_{*}^{4}}{256} \approx-\frac{15 \gamma^{2}}{16 k^{2}}$ of order $\varepsilon^{2}$.

For $k=4, \gamma-0,05$ we have $a_{0}^{2}=1, a_{*}^{2} \approx 0.97$.

Remark. The quadratic nonolinearity $\beta x^{2}$ of order $\varepsilon$ has no effect on the amplitude; it introduces only a negligible additional frequency of order $\varepsilon^{2}$ (and also a perturbation of order $\varepsilon$ ).

\section{§2. The cubic non-linearity and an external excitation of order $\varepsilon$}

For non autonomous systems, we first consider a forced one with two elements of order $\varepsilon$ : - the cubic non-linearity and - an external excitation in the subharmonic resonance of order one - third. This is the only case in which the interaction between the cubic non-linearity and external excitations of order $\varepsilon$ (in non principal resonances) may occur and, as result, we obtain in the second approximation, a quadratic parametric excitation. The differential equation governing the system under consideration is of the form:

$$
\ddot{x}+\omega^{2} x=\varepsilon\left\{\Delta x-\gamma x^{3}-8 e \omega^{2} \cos 3 \omega t\right\}-\varepsilon^{2} h \dot{x},
$$

where $8 e>0,3 \omega$ are intensity and frequency of the external excitation of interest; $\varepsilon \Delta=\omega^{2}-1$ is the detuning parameter; $k>0$ is the damping coefficient assumed to be order $\varepsilon^{2}$; other notations have been given in $\S 1$. In the first approximation, the asymptotic solution is of the form:

$$
\begin{array}{ll}
x=a \cos \psi, & \psi=\omega t+\theta, \\
\dot{a}=\varepsilon A_{1}(a, \theta), & \dot{\theta}=\varepsilon B_{1}(a, \theta) .
\end{array}
$$

Substituting (2.2) into (2.1), using (2.3), equating the terms of like power in $\varepsilon$ then the ones of same harmonics in $\psi$ we obtain:

$$
\dot{a}=\varepsilon A_{1}(a, \theta), \quad \dot{\theta}=\varepsilon B_{1}(a, \theta)=\frac{-\varepsilon}{2 \omega}\left(\Delta-\frac{3 \gamma}{4} a^{2}\right)
$$

We have thus a family of harmonic oscillations with arbitrary constant amplitude $a_{0}$ and initial dephase $\theta_{0}$ and with frequency

$$
\dot{\psi}=\omega+\dot{\theta}=\omega-\frac{\varepsilon}{2 \omega}\left(\Delta-\frac{3 \gamma}{4} a_{0}^{2}\right)
$$

the latter depends on $a_{0}$. 
In the second approximation, the asymptotic solution is given by

$$
\begin{gathered}
x=a \cos \psi+\varepsilon u_{1}(a, \psi, \theta)=a \cos \psi=\varepsilon\left\{\frac{\gamma a^{3}}{32} \cos 3 \psi+e \cos (2 \psi-3 \theta)\right\} \\
\left\{\begin{array}{c}
\dot{a}=f=\varepsilon^{2} A_{2}(a, \theta)=\frac{-\varepsilon^{2} a}{2 \omega}\left\{h \omega-\frac{3 \gamma e}{4} a \sin 3 \theta\right\}, \\
\dot{\theta}=g=\varepsilon B_{1}(a, \theta)+\varepsilon^{2} B_{2}(a, \theta)= \\
=\frac{-\varepsilon}{2 \omega}\left(\Delta-\frac{3 \gamma}{4} a^{2}\right)-\frac{\varepsilon^{2}}{2 \omega}\left\{B_{1}^{2}-\frac{3 \gamma^{2}}{128} a^{4}-\frac{3 \gamma e}{4} a \cos 3 \theta\right\} .
\end{array}\right.
\end{gathered}
$$

Constant amplitude and dephase of stationary oscillations satisfy the equations:

$$
f=0, \quad g=0 .
$$

From the second equation, it follows that $B_{1}$ i.e. $\left(\Delta-\frac{3 \gamma}{4} a^{2}\right)$ must be of order $\varepsilon^{2}$. So, we can neglect $B_{1}^{2}$ and rewrite $(2.2)$ as:

$$
\left\{\begin{array}{l}
h \omega-\frac{3 \gamma e}{4} a \sin 3 \theta=0, \\
\left(\Delta-\frac{3 \gamma}{4} a^{2}-\frac{3 \gamma^{2}}{128} a^{4}\right)-\frac{3 \gamma e}{4} a \cos 3 \theta=0 .
\end{array}\right.
$$

It is easy to see that in the second approximation the interaction between the cubic non-linearity and the external excitation of frequency $3 \omega$ produces a quadratic parametric excitation of order $\varepsilon^{2}$ which is represented by the terms

$$
\left(-\frac{3 \gamma e}{4} a \sin 3 \theta,-\frac{3 \gamma e}{4} a \cos 3 \theta\right) \text {. }
$$

Other effects of the cubic non-linearity are: - a perturbation $\frac{\gamma a^{3}}{32} \cos 3 \psi$ of order $\varepsilon$ and - a negligible additional frequency $-\frac{3 \gamma^{2}}{128} a^{4}$ of order $\varepsilon^{2}$.

In the plane $\left(\Delta, a^{2}\right)$, the resonance curve can be identified by the frequency amplitude relationship:

$$
W\left(\Delta, a^{2}\right)=\left(\Delta-\frac{3 \gamma}{4} a^{2}-\frac{3 \gamma^{2}}{128} a^{4}\right)^{2}+h^{2} \omega^{2}-\frac{9 \gamma^{2}}{16} e^{2} a^{2}=0,
$$

or approximatively

$$
\delta=\frac{3 \gamma}{4} a^{2}+\frac{3 \gamma^{2}}{128} a^{4} \pm \sqrt{\frac{9 \gamma^{2}}{16} e^{2} a^{2}-h^{2}}
$$


For fixed given parameters $\gamma=0.16, e=0.05$, the resonance curves (0), (1) in Fig. 1 correspond to $h=0.000,0.003$ respectively. The two conditions of stability

$$
h>0, \quad \frac{\partial W}{\partial a^{2}}>0
$$

are satisfied for the system with damping and along the upper branch of the resonance curve

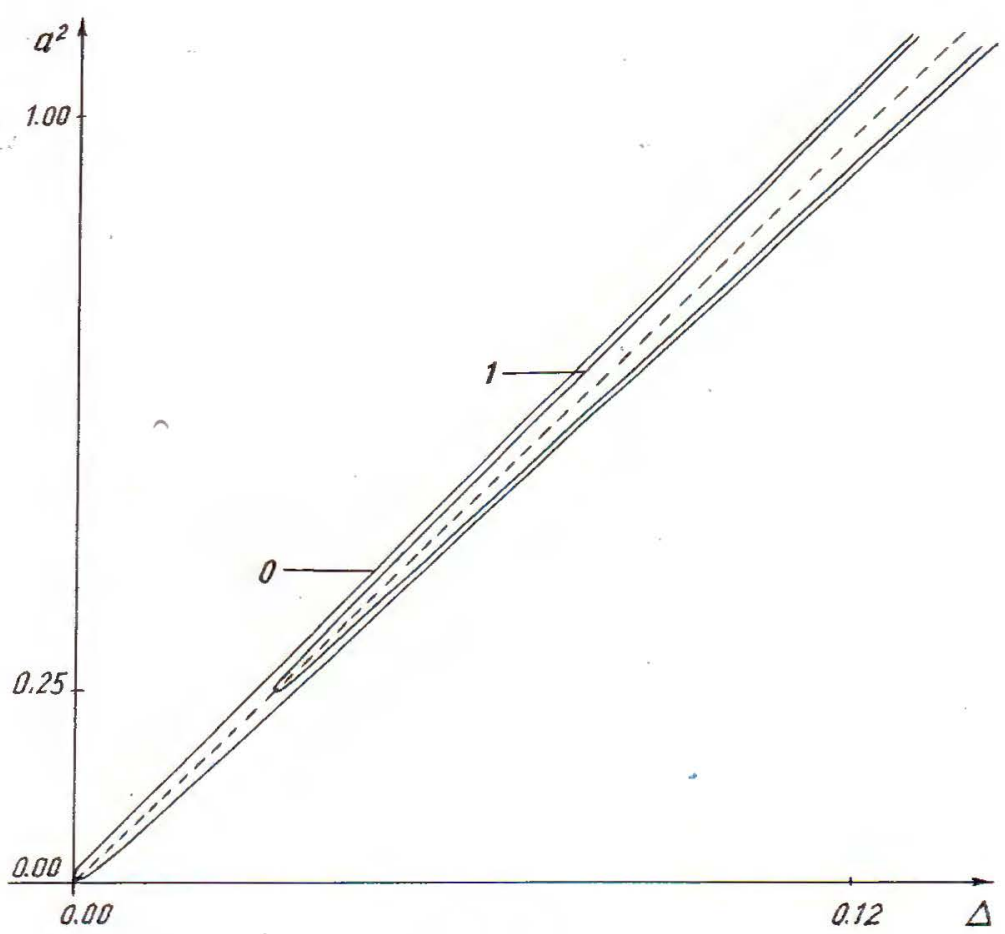

Fig. 1

Remark 1. In the second approximation, the cubic non-linearity does not interact with external excitations $e_{n} \cos n \omega t$ if $n \neq 1,3$.

Remark 2. Although $\left(\Delta-\frac{3 \gamma}{4} a^{2}\right)$ is of order $\varepsilon^{2}$, the cubic non-linearity coefficient $\gamma$ and the detuning parameter $\delta$ may be a little large and the resonance region may be of order $\varepsilon$. In the case of the quadratic non-linearity, the resonance region is very narrow (of order $\varepsilon^{2}$ ).

\section{§3. The cubic non-linearity and parametric excitations of order $\varepsilon$}

The second type of non-autonomous systems is that of parametrically - excited ones. For this type, the cubic non-linearity may interact with different parametric excitations. As an illustration, let us consider a system described by the differential 
equation:

$$
\ddot{x}+\omega^{2} x=\varepsilon\left\{\Delta x-\gamma x^{3}+2 p x \cos 4 \omega t\right\}-\varepsilon^{2} h \dot{x},
$$

where: $2 p>0$ and $4 \omega$ are intensity and frequency of the parametric excitation interested (which belongs to the type $2 p x^{m} \cos m \omega t,|n-m|=3$ ), other notations have been explained in $\S 2$.

In the first approximation, we have the same family of harmonic oscillations as that corresponding in $\S 2$.

In the second approximation, the asymptotic solution is given by:

$$
\begin{gathered}
x=a \cos \psi+\varepsilon u_{1}(a, \psi, \theta)= \\
=a \cos \psi+\varepsilon\left\{\frac{\gamma a^{3}}{32} \cos 3 \psi+\frac{p a}{8} \cos (3 \psi-4 \theta)+\frac{p a}{24} \cos (5 \psi-4 \theta)\right\} \\
\left\{\begin{aligned}
\dot{a}= & \varepsilon^{2} A_{2}(a, \theta)=\frac{-\varepsilon^{2}}{2 \omega}\left\{h \omega-\frac{p \gamma a^{2}}{32} \sin \theta+\frac{p^{2}}{12} \sin 3 \theta-\frac{3 p \gamma a^{2}}{16} \sin 4 \theta\right\} \\
\dot{\theta}= & \varepsilon B_{1}(a, \theta)+\varepsilon^{2} B_{2}(a, \theta)=\frac{-\varepsilon}{2 \omega}\left(\Delta-\frac{3 \gamma}{4} a^{2}\right) \\
& -\frac{\varepsilon^{2}}{2 \omega}\left\{B_{1}^{2}-\frac{3 \gamma^{2}}{128} a^{4}-\frac{p \gamma a^{2}}{32} \cos \theta-\frac{p^{2}}{6} \cos 3 \theta-\frac{3 p \gamma a^{2}}{16} \cos 4 \theta\right\}
\end{aligned}\right.
\end{gathered}
$$

Neglecting $B_{1}^{2}$, we write the equations for stationary oscillations as:

$$
\left\{\begin{array}{l}
h \omega-\frac{p \gamma a^{2}}{32} \sin \theta+\frac{p^{2}}{12} \sin 3 \theta-\frac{3 p \gamma a^{2}}{12} \sin 4 \theta=0, \\
\left(\Delta-\frac{3 \gamma}{4} a^{2}-\frac{3 \gamma^{2}}{128} a^{4}\right)-\frac{p \gamma a^{2}}{32} \cos \theta-\frac{p^{2}}{6} \cos 3 \theta-\frac{3 p \gamma a^{2}}{16} \cos 4 \theta=0 .
\end{array}\right.
$$

Do not identifying the resonance curve, we note only that, in the second approximation, beside the excitation $\left(\frac{p^{2}}{12} \sin 3 \theta,-\frac{p^{2}}{6} \cos 3 \theta\right)$ due to the parametric excitation, there are also two excitations

$$
\left(-\frac{p \gamma a^{2}}{32} \sin \theta,-\frac{p \gamma a^{2}}{32} \cos \theta\right) \text { and }\left(-\frac{3 p \gamma a^{2}}{16} \sin 4 \theta,-\frac{3 p \gamma a^{2}}{16} \cos 4 \theta\right)
$$

due to the interaction between the cubic non-linearity and the aforesaid parametric excitation of order $\varepsilon$.

\section{§4. The cubic non-linearity and excitations of order $\varepsilon^{2}$}

In this last section, we examine the interaction between the cubic non-linearity (and other elements) of order $\varepsilon$ with excitations of order $\varepsilon^{2}$. As and illustration we 
consider a combination of two system (1.1) (2.1), assuming that the self-excitation is of order $\varepsilon^{2}$ :

$$
\ddot{x}+\omega^{2} x=\varepsilon\left\{\Delta x-\gamma x^{3}-8 e \omega^{2} \cos 3 \omega t\right\}+\varepsilon^{2} h(1-k x) \dot{x} .
$$

In the second approximation, the asymptotic solution is determined from:

$$
\begin{gathered}
x=a \cos \psi+\varepsilon u_{1}(a, \psi, \theta)=a \cos \psi+\varepsilon\left\{\frac{\gamma a^{3}}{32} \cos 3 \psi=e \cos (3 \psi-3 \theta)\right\}, \\
\left\{\begin{array}{c}
\dot{a}=f=\varepsilon^{2} A_{2}(a, \theta)=\frac{\varepsilon^{2} a}{2 \omega}\left\{h \omega\left(1-\frac{k a^{2}}{4}\right)+\frac{3 \gamma e}{4} a \sin 3 \theta\right\} \\
\dot{\theta}=g=\varepsilon B_{1}(a, \theta)+\varepsilon^{2} B_{2}(a, \theta)= \\
=-\frac{\varepsilon}{2 \omega}\left(\Delta-\frac{3 \gamma}{4} a^{2}\right)-\frac{\varepsilon^{2}}{2 \omega}\left\{B_{1}^{2}-\frac{3 \gamma^{2}}{128} a^{4}-\frac{3 \gamma e}{4} a \cos 3 \theta\right\} .
\end{array}\right.
\end{gathered}
$$

Neglecting $B_{1}^{2}$ (which are of order $\varepsilon^{4}$ ) we write the equations for stationary oscillations as:

$$
\left\{\begin{array}{l}
h \omega\left(1-\frac{k}{4} a^{2}\right)+\frac{3 \gamma e}{4} a \sin 3 \theta \\
\left(\Delta-\frac{3 \gamma}{4} a^{2}-\frac{3 \gamma^{2}}{128} a^{4}\right)-\frac{3 \gamma e}{4} a \cos 3 \theta=0
\end{array}\right.
$$

If $e=0$ (without excitation of order $\varepsilon$ ), beside the perturbation $\frac{\gamma a^{3}}{32} \cos 3 \psi$ (of order $\varepsilon$ ), the cubic non-linearity introduces only a negligible additional frequency $\frac{-3 \gamma^{2} a^{4}}{128}$ (of order $\varepsilon^{2}$ ), the amplitude $a^{2}=\frac{4}{k}$ of stationary oscillations is fully determined by the self-excitation (of order $\varepsilon^{2}$ ). Thus, for systems with excitations of order $\varepsilon^{2}$, the effect of the "single" cubic non-linearity in the second approximation is very weak.

If $e \neq 0$ (with excitation of order $\varepsilon$ ) it appears a parametric excitation, represented by the terms $\left(\frac{3 \gamma e}{4} a \sin 3 \theta,-\frac{3 \gamma e}{4} a \cos 3 \theta\right)$, due to the interaction between the cubic non-linearity and the external excitation of order $\varepsilon$ and the system will oscillate in "combined" regime "parametrically and self-excited one". The resonance curve of this regime can be identified by the frequency amplitude relationship

$$
W\left(\Delta, a^{2}\right)=\left(\Delta-\frac{3 \gamma}{4} a^{2}-\frac{3 \gamma^{2}}{128} a^{4}\right)^{2}+h^{2} \omega^{2}\left(1-\frac{k a^{2}}{4}\right)^{2}-\frac{9 \gamma^{2} e^{2} a^{2}}{16}=0
$$

or, approximatively

$$
\Delta=\frac{3 \gamma}{4} a^{2}+\frac{3 \gamma^{2}}{128} a^{4} \pm \sqrt{\frac{9 \gamma^{2} e^{2}}{16} a^{2}-h^{2}\left(1-\frac{k}{4} a^{2}\right)^{2}}
$$


For fixed given parameters $\gamma=0.16, e=0.05, k=4$, the resonance curves (0), (1), (2) shown in Fig. 2 correspond to $h=0.001,0.005$ respectively. We see that the resonance curve (with admissible amplitudes) is of parametric character (since $h$ is very small - of order $\varepsilon^{2}$ ). Increasing $h$, the resonance curve is contracted, it takes the form of an "oval", encircling the point $\mathrm{I}\left(\Delta=\frac{3 \gamma}{4}=0.12, a^{2} \approx \frac{4}{k}=1\right)$ the latter is the representative point of the purely self-excited oscillation.

Stable stationary oscillations correspond to the upper branch of the resonance curve.

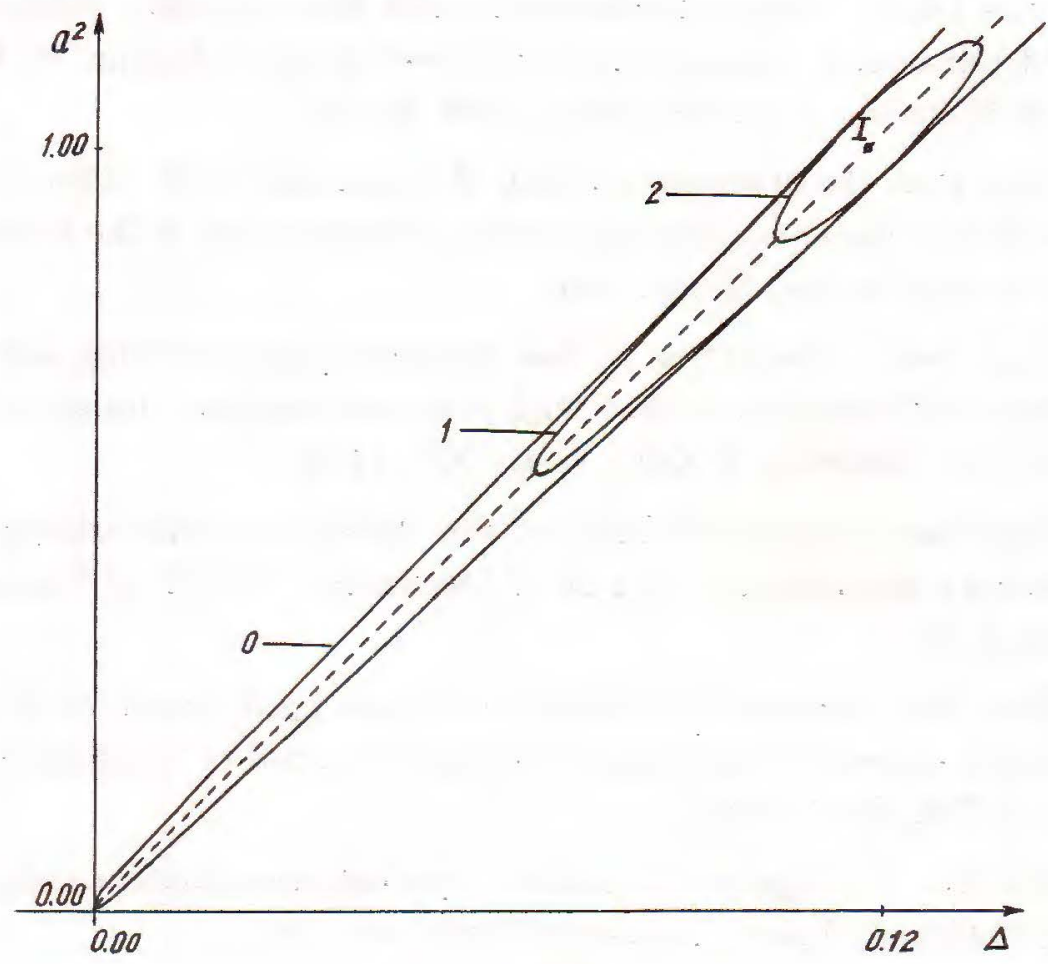

Fig. 2

Conclusion.

We have examined some quasi-linear systems with the cubic restoring nonlinearity of order $\varepsilon$, In the first approximation, we have a family of harmonic oscillations whose frequency depends on the cubic nonlinearity coefficient. In the second approximation, the interaction between the non-linearity interested with certain types of excitation may cause different effects.

This publication is completed with financial support from The Council for Natural Sciences of Vietnam. 


\section{REFERENCES}

1. Nayfeh A. H. Interaction of fundamental parametric resonances with subharmonic resonances of order one-half. Journal of Sound and Vibration 96 (3) (1984), 233-240.

2. Nguyen Van Dinh. Resonance in a quasilinear system under two parametric excitations. Proceedings of the Fifth National Conference on Mechanics. Hanoi, 1993.

3. Nguyen Van Dinh. Nonlinearities in a quasi-linear system subjected to external and parametric excitations of different orders. Journal of Mechanics, NCNST of Vietnam, T. XVIII, No 1, 1996, 22-26.

4. Nguyen Van Dao. Interaction between the elements with different orders of smallness in non-linear oscillating systems. Proceedings of the Sixth National Congress on Mechanics. Hanoi, 1997.

5. Nguyen Van Dao. Interaction of the elements characterizing the quadratic nonlinearity and forced excitation with other excitations. Journal of Mechanics, NCNST of Vietnam, T.XIX, No4, 1997, 11-20.

6. Nguyen Van Dao. Interaction between the elements characterizing the forced and parametric excitations. Journal of Mechanics NCNST of Vietnam T.XX, No $1,1998,9-20$.

7. Nguyen Van Dao. Interaction between the forced and parametric excitations with different degrees of smallness, Vietnam Journal of Mechanics, NCST of Vietnam, T. XX, No 2, 1998, 11-17.

8. Mitropolski Yu. A., Nguyen Van Dao. Applied asymptotic methods in nonlinear oscillations, Kluwer Academic Publisher, 1997.

Received September 15, 1998

\section{PHI TUYẾN BẬC BA CẤP $\varepsilon$ Ở XẨP Xİ THỨ HAI}

Xét một số hệ dao động á tuyến có số hạng hồi phục phi tuyến bậc ba ớ cấp $\varepsilon$ ̛̛ xấp xî thứ hai. Ờ xấp xỉ thứ nhất, có họ dao động tuần hoàn với tần số phụ thuộc số hạng phi tuyến bậc ba. Ở xấp xỉ thứ hai, tương tác giữa số hạng phi tuyến nói trên là một số loại kích động cùng cấp $\varepsilon$ có thể gây ra nhiều hiệu ứng khác nhau. 